\title{
Oral neuromuscular training in patients with dysphagia after stroke: a prospective, randomized, open-label study with blinded evaluators
}

\author{
Patricia Hägglund ${ }^{1,2^{*}+}$ C, Mary Hägg ${ }^{3,4 \dagger}$, Eva Levring Jäghagen ${ }^{1}$, Bengt Larsson ${ }^{5}$ and Per Wester ${ }^{2,6}$
}

\begin{abstract}
Background: Oral and pharyngeal swallowing dysfunction are common complications in acute stroke patients. This primary aim of this study was to determine whether oral neuromuscular training improves swallowing function in participants with swallowing dysfunction after stroke. A secondary aim was to assess how well results of the timed water-swallow test (TWST) correspond with swallowing dysfunction diagnosed by videofluoroscopy (VFS).

Methods: This was an intention-to-treat two-centre prospective randomized open-label study with blindedevaluators (PROBE) design. At 4 weeks after stroke onset, participants with swallowing dysfunction were randomized to 5 weeks of continued orofacial sensory-vibration stimulation with an electric toothbrush or additional oral neuromuscular training with an oral device $\left(M_{\text {uppy }}{ }^{\oplus}\right)$. Participants were examined with TWST, a lip-force test, and VFS before (baseline), after 5 weeks' treatment (the end-of-treatment), and 12 months after treatment (follow-up). The baseline VFS results were compared with the TWST results. The primary endpoint was changes in swallowing rate assessed using TWST, from baseline to the end of training and from baseline to follow-up based on intentionto-treat analyses. The secondary endpoint was the corresponding changes in lip-force between baseline, the end of treatment, and follow-up.
\end{abstract}

Results: The participants were randomly assigned as controls $(n=20)$ or for intervention with oral neuromuscular training $(n=20)$. After treatment, both groups had improved significantly (intervention, $P<0.001$; controls, $P=0.001$ ) in TWST but there was no significant between-group difference in swallowing rate. At the 12-month follow-up, the intervention group had improved further whereas the controls had deteriorated, and there were significant between-group differences in swallowing rate $(P=0.032)$ and lip force $(P=0.001)$. A TWST $<10 \mathrm{~mL} / \mathrm{sec}$ at baseline corresponded to VFS-verified swallowing dysfunction in all assessed participants.

(Continued on next page)

\footnotetext{
* Correspondence: patricia.hagglund@umu.se

${ }^{+}$Hägglund Patricia and Hägg Mary contributed equally to this work.

'Department of Odontology, Oral and Maxillofacial Radiology, Umeå University, SE-90187 Umeå, Sweden

${ }^{2}$ Umeå Stroke Center, Department of Public Health and Clinical Medicine, Umeå University, Umeå, Sweden

Full list of author information is available at the end of the article
}

(c) The Author(s). 2020 Open Access This article is licensed under a Creative Commons Attribution 4.0 International License, which permits use, sharing, adaptation, distribution and reproduction in any medium or format, as long as you give appropriate credit to the original author(s) and the source, provide a link to the Creative Commons licence, and indicate if changes were made. The images or other third party material in this article are included in the article's Creative Commons licence, unless indicated otherwise in a credit line to the material. If material is not included in the article's Creative Commons licence and your intended use is not permitted by statutory regulation or exceeds the permitted use, you will need to obtain permission directly from the copyright holder. To view a copy of this licence, visit http://creativecommons.org/licenses/by/4.0/ The Creative Commons Public Domain Dedication waiver (http://creativecommons.org/publicdomain/zero/1.0/) applies to the data made available in this article, unless otherwise stated in a credit line to the data. 
(Continued from previous page)

Conclusion: The 5-week oral neuromuscular training improved swallowing function in participants with post-stroke dysphagia compared with the controls 12 months after intervention, but there was no between-group difference in improvement immediately after treatment. TWST results corresponded with VFS results, making TWST a feasible method for identifying persons with swallowing dysfunction after stroke. Larger randomized controlled trials are required to confirm our preliminary positive long-term results.

Trial registration: Retrospectively registered at ClinicalTrials.gov: NCT04164420. Registered on 15 November 2019.

Keywords: Swallowing disorder, Oral screen, Rehabilitation, Swallowing capacity, Videofluoroscopy, Radiology

\section{Background}

Oropharyngeal dysphagia (symptoms of swallowing dysfunction related to the oral cavity and/or pharynx) is one of the most common complications in acute stroke [1]. Traditionally, dysphagia has been managed by compensatory strategies: for example, postural adjustments, swallowing manoeuvres, or modifying the consistency of food/liquid. However, during the late 1990s, there was increasing interest in caring for and improving swallowing function in stroke patients with dysphagia. Orofacial regulation therapy was introduced in 1990 [2], especially as therapy for children with oropharyngeal dysphagia. It comprises orofacial sensory-vibration stimulation with an electric toothbrush [3] or manual massage, sometimes in combination with active sensorimotor training with various oral appliances such as a palatal plate [4] or an oral neuromuscular training device [5]. In 2008, the Muppy ${ }^{\oplus}$ oral device (see Fig. 1) was used to measure lip force with a newton meter (see Fig. 2), and the study showed a significant difference in lip force between healthy subjects and stroke-affected patients with swallowing dysfunction [6]. Lip force and swallowing function are shown to depend on the same complex neuromuscular activity [7]. The same activity that initiates a swallow is activated when lip-force is measured [8]. This suggested that patients with dysphagia could use an oral device to self-train their weak oral and pharyngeal muscles; that is, for neuromuscular training. Improvement in swallowing function has been reported among older people with dysphagia after oral

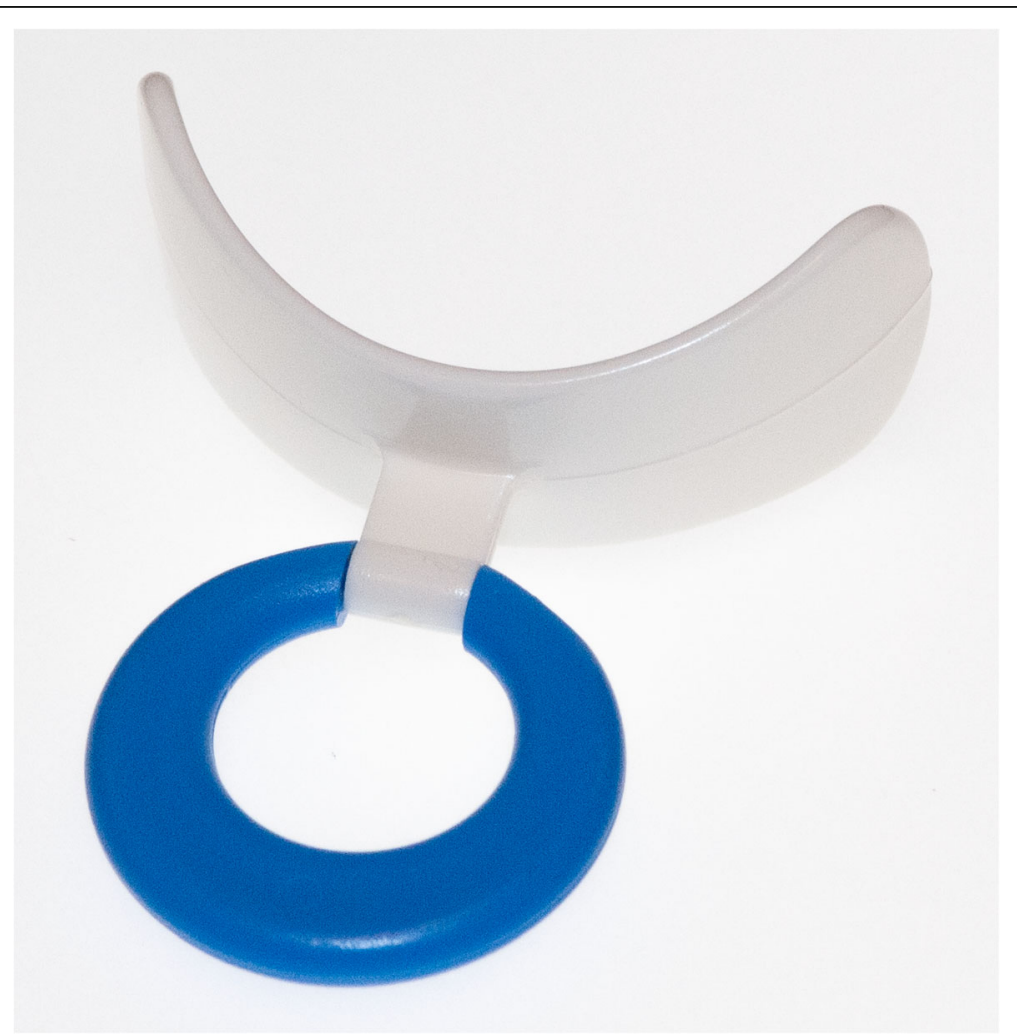

Fig. 1 The oral device $\left(\right.$ Muppy $^{\oplus}$ ) (own image) 


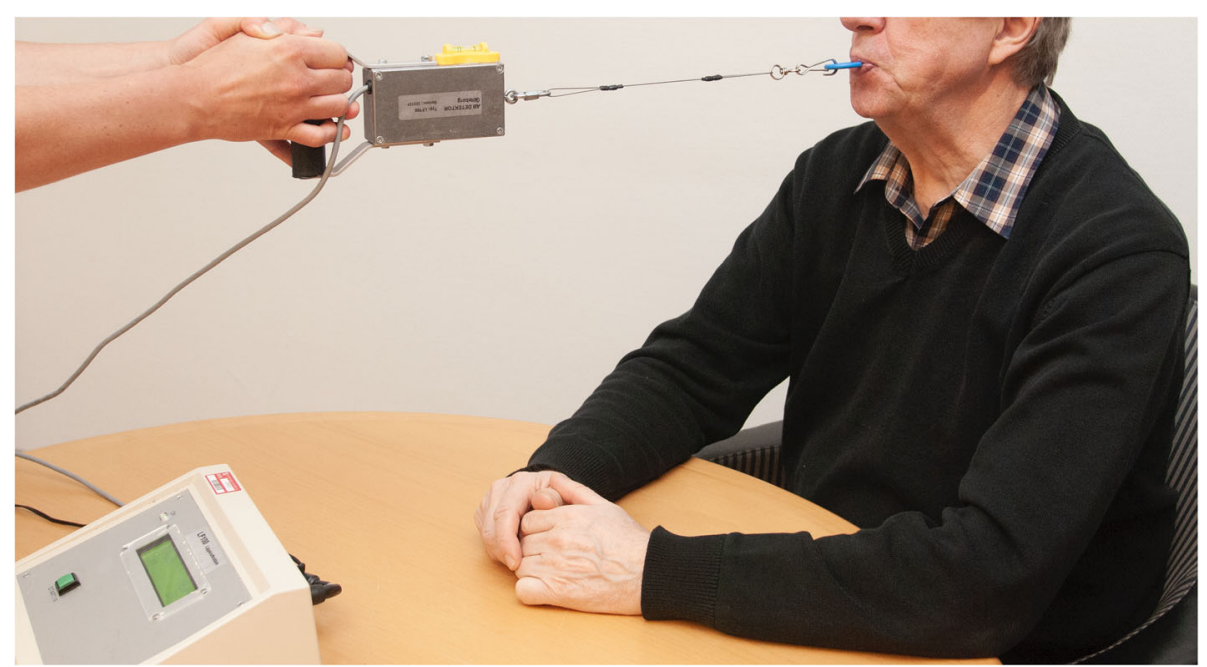

Fig. 2 The lip force meter, LF100 (MHC1 AB Detector, Gothenburg, Sweden) (own image). LF100 is a device for measuring the force (in newtons) of the buccinators. The handle on the LF100 is connected to the oral device

neuromuscular training [9]. Until now, no randomized, controlled study has been performed to investigate the effect of oral neuromuscular training as treatment for post-stroke dysphagia. This study aimed to determine whether adding oral neuromuscular training to orofacial sensory-vibration stimulation improves swallowing function in participants with swallowing dysfunction after a stroke. The secondary aim was to assess how well the results of the timed water-swallow test (TWST) [10-12] correspond with findings of swallowing dysfunction from videofluoroscopy (VFS).

\section{Methods}

\section{Study design and setting}

This was a two-centre study with an intention-to-treat, prospective, randomized, open-label trial with blinded evaluators (PROBE) design. Information on the study design can be found at ClinicalTrials.gov, identifier: NCT04164420. The study was conducted according to the recommendations for Interventional Trials and the CONSORT guidelines for randomised controlled trials (Additional file 1). Patients with a first-ever stroke and persistent swallowing dysfunction 4 weeks post-stroke according to the timed water-swallow test (TWST) [10-12], treated at Umeå University Hospital or Hudiksvall Hospital, Sweden, were offered to participate in the interventional study. The participants were randomized into an intervention group that received oral neuromuscular training for 5 weeks or a control group. A randomization list was constructed by the statistician and trial manager and was stored in envelopes locked in a cupboard. The randomization list did not include any stratification or minimization. For ethical reasons, both groups continued to receive orofacial sensory-vibration stimulation in parallel, initiated before inclusion, if the patients showed dysphagia 1 week after their stroke. Evaluations were made at baseline (4 weeks after stroke onset), at the end of treatment (after 5 weeks treatment), and at the 12-month follow-up after a period without treatment to determine if any lasting positive training effect was present. At the end of treatment and 12-month follow-up, the evaluating assessors were blinded to what treatment the participants had received.

\section{Participants}

Inclusion criteria for participation were the following: first-time stroke and a pathological TWST [10-12] 4 weeks after stroke onset. Exclusion criteria were: inability to cooperate, percutaneous endoscopic gastrostomy (PEG), neurological diseases other than stroke, known history of dysphagia prior to the stroke, prominent horizontal overbite (contra-indication due to the oral device's design), or hypersensitivity to the acrylate.

\section{Treatment}

\section{Oral neuromuscular training}

An oral device (Muppy'; Dr. Hinz Dental, Herne, Germany) was used for oral neuromuscular training (see Fig. 1) that aims to stimulate sensory input and strengthen the facial, oral, and pharyngeal muscles [13]. During the training, the device was placed pre-dentally behind closed lips, and the patient sat with the body and head in a strictly upright position. The participants were instructed to hold the device against a graduallyincreasing horizontal pulling force for 5-10 s, whilst trying to resist the force by tightening the lips [13]. The participants were instructed to achieve a pulling force that was as high as possible without losing hold of the 
device with the lips. The exercise was performed three times per session, and three times daily before eating. If the participant was unable to hold the oral device, relatives or ward staff were instructed to assist in providing traction at a right angle to the mouth. Training was also possible when sitting in a bed with the head supported firmly. Verbal, practical, and written instructions about training at home were given to the participants, relatives, or care assistants. The participant or care giver reported the training in a log book that was evaluated after a 5week training period. The log book was placed visible for the participant and care givers as a reminder of keeping up the training, and it was considered reliable.

\section{Orofacial sensory-vibration stimulation}

All participants in the intervention group and the control group self-administered or were assisted by relatives or ward staff in orofacial sensory-vibration stimulation (see Fig. 3) by using an electric toothbrush with a round and rotating head (Oral- $\mathrm{B}^{\circ}$, Procter \& Gamble, Cincinnati, USA) [3]. Instructions were given on how to stimulate the buccinator mechanism [4, 7], lips, external floor, and the tongue, three times daily before a meal.

\section{Outcome}

The primary outcome was changes in swallowing rate measured by TWST [10-12] at the end of treatment compared with baseline and 12 months post-treatment, within each group and comparing the intervention group with the control group. Secondary outcomes were changes in lip force measured by a lip-force test [6] at the end of treatment compared with baseline and 12 months post-treatment, comparing the intervention group with the control group. Swallowing dysfunction according to VFS, including the presence of aspirationpenetration as sign of unsafe swallowing as well as premature spillage and pharyngeal residue as signs of ineffective swallowing, was assessed for comparison with the TWST results.

\section{Procedure}

For both study groups, the swallowing rate (TWST), lip force, and swallowing function (VFS) were assessed. A nurse, speech-and-language pathologist, or physician performed the assessments, except the VFS examinations, which were performed by a general or an oraland-maxillofacial radiologist. A flow-chart of the included participants and the study process is shown in Fig. 4.

\section{Timed Water-Swallow Test (TWST)}

The participant first received a teaspoon of water, repeated three times consecutively. In case of a total inability to swallow, or if a swallow was misdirected (e.g., there were clinical signs of aspiration such as cough or wet/gurgly voice), the swallowing rate was graded as zero and the TWST was not performed. If the swallowing was safe during the teaspoon test, a TWST was completed.

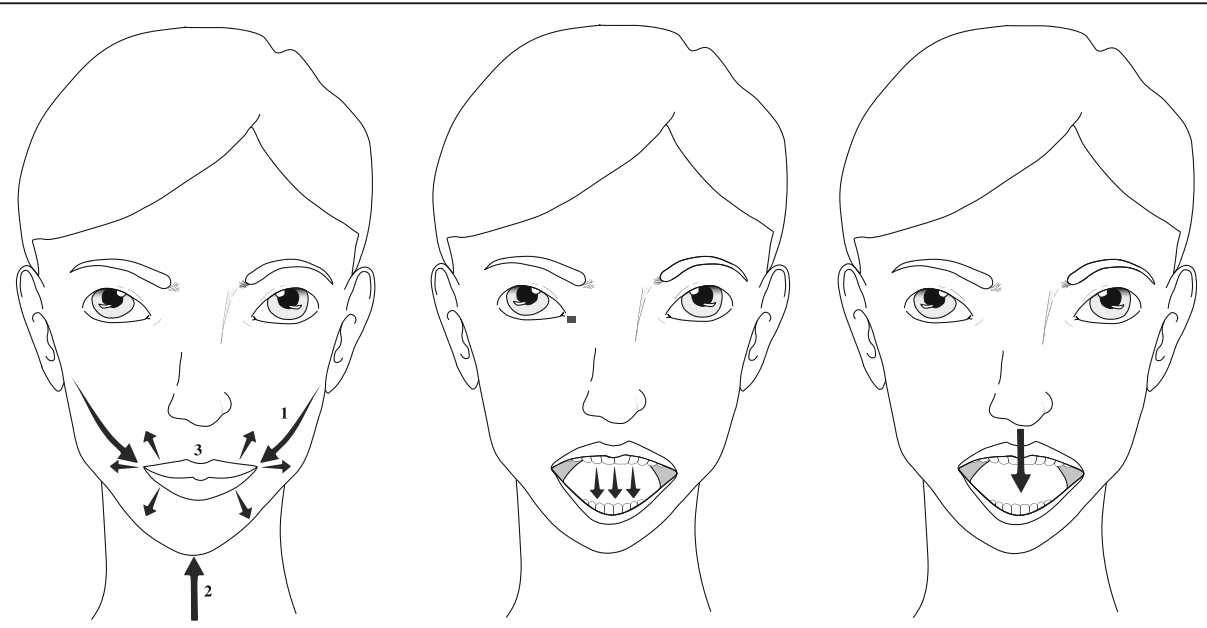

Fig. 3 Orofacial sensory-vibration stimulation using an electric toothbrush. (Left) Stimulation of (1) the buccinator mechanism that activates tongue retraction; (2) the muscles of the floor ( $\mathrm{m}$. digastricus anterior abdomen, $\mathrm{m}$. mylohyoideus and $\mathrm{m}$. geniohyoideus) that will lift the hyoid bone forward and upward and activate the swallow reflex; and (3) the lips, enhancing lip closure. (Middle) Stimulation of the front of the tongue that activates and raises the root of the tongue, thus passively activating the receptors in the anterior faucial arch to perform the swallowing reflex and indirectly lift the velum. (Right) Stimulation of the tip of the tongue by downward pressure improves the force of the tongue. All the different movements in the left, middle, and right images also provide sensory stimulation (through $\mathrm{n}$ trigeminus, afferent pathway) and feedback as a motor response (through $\mathrm{n}$ facialis, efferent pathway), that describe the sensory-motor reflex arc. Illustrations: Anna Jäghagen (own illustration/image) 


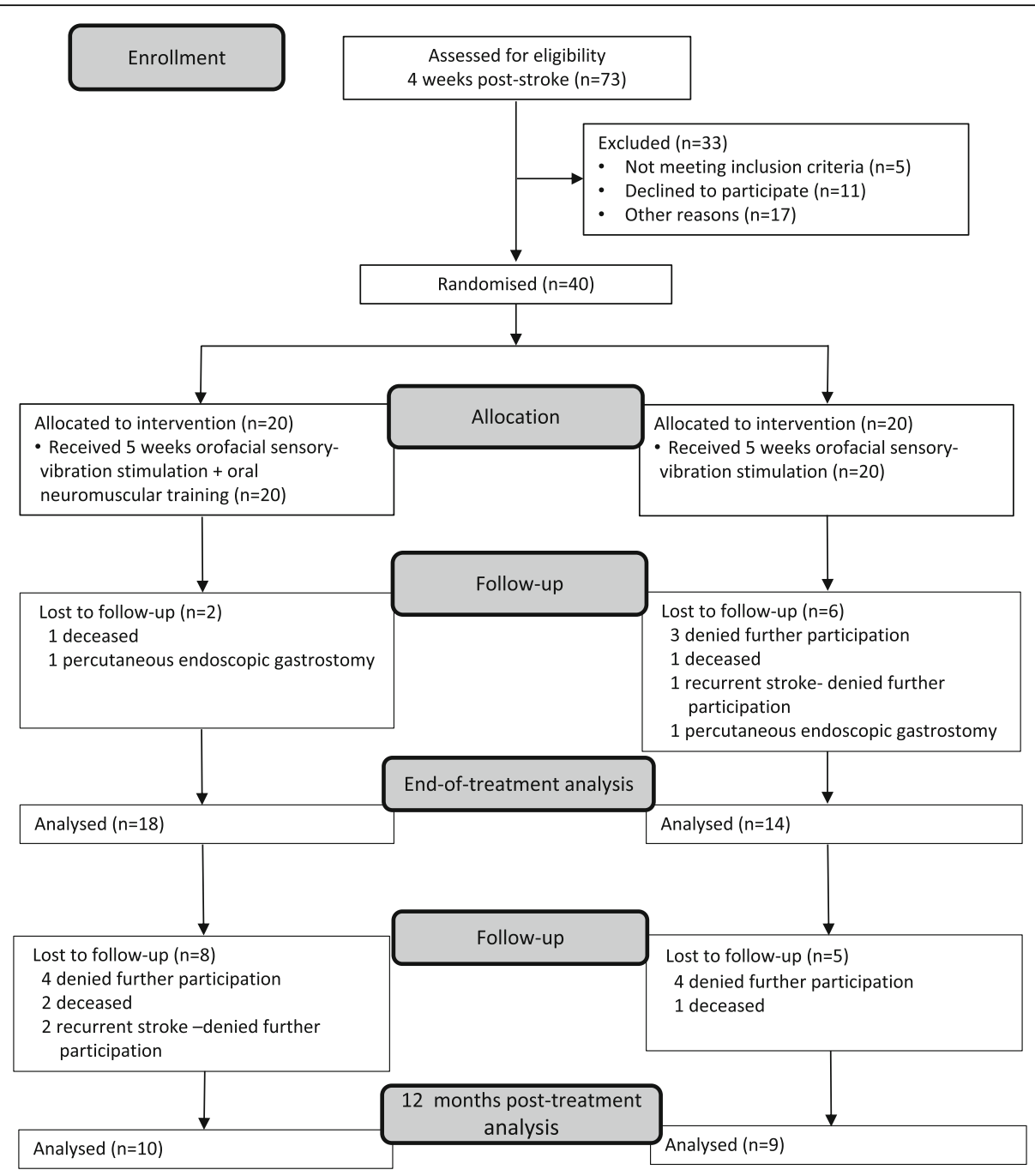

Fig. 4 Flowchart of the subject inclusion and data collection processes

The TWST is a timed test of a person's ability to swallow water [10-12]. It was performed with the participants sitting in upright position. The participants were instructed to swallow water $(150 \mathrm{~mL})$ as quickly as possible without pausing. The time was recorded from the start of drinking until the last swallow was completed. Any remaining water in the glass was measured. The swallowing rate was calculated as the amount of swallowed water (in millilitres) divided by time (seconds). A swallowing rate of $10 \mathrm{~mL} / \mathrm{s}$ was regarded as the lower normal limit [10-12]. The TWST has been demonstrated to have high intra-rater, inter-rater, and testretest reliability and to be a valid index for assessing swallowing dysfunction [10-12, 14].

\section{Lip-force test}

Lip force was recorded in newtons using a LF100 (MHC1 AB Detector, Gothenburg, Sweden) [6] (see Fig. 2). The patient was instructed to hold a pre-dentally placed oral device (Muppy ${ }^{\circ}$ ) (see Fig. 1) for as long as possible by tightening the lips and pressing the head backwards as the investigator applied traction at a right angle to the patient's mouth with increasing force for $10 \mathrm{~s}$, or until the subject lost their grip of the oral device. Each subject was measured three times, with a 2-min rest between attempts. The maximum lip-force value was recorded and blinded for the investigator. The lower limit for normal lip force was $15 \mathrm{~N}$ [6].

\section{Videofluoroscopy (VFS)}

During VFS in lateral projection, the participant initially swallowed $5 \mathrm{~mL}$ of iodine contrast medium (Visipaque $320 \mathrm{mg} \mathrm{I} / \mathrm{mL}$ ) delivered with a spoon, to assess the presence of aspiration (before, during, or after swallowing). If severe aspiration was present, the examination was concluded. If no or minor aspiration was found, a bolus 
of $10 \mathrm{~mL}$ iodine contrast medium was tested. If there was no aspiration, the examination proceeded with 10 and $20 \mathrm{~mL}$ liquid barium sulphate contrast medium (Mixobar ${ }^{\circ}$ Colon $1 \mathrm{mg} / \mathrm{mL}$ ) delivered with a spoon, and a free amount of liquid barium sulphate from a cup. If the patient was still free of aspiration, this was followed by assessment in the frontal projection with $5 \mathrm{~mL}, 20$ $\mathrm{mL}$, and a free amount of barium sulphate contrast medium. To assess correspondence with TWST, oral and pharyngeal residue (bolus retention after the bolus has passed through the pharynx), premature spillage (bolus entering the pharynx without eliciting the swallowing reflex, with the airway remaining open with increased risk of aspiration), penetration (bolus entering the larynx and proceeding below the vocal cords), and aspiration (bolus entering the airway below the vocal cords) with and without cough were recorded. The improvement in swallowing function was assessed according to the penetration-aspiration scale (the PAS is scored 1-8, where score 1 means no bolus enters the airway, and scores 2-8 are different degrees of penetration or aspiration into the airway) [15]. The PAS scores were dichotomised into $\leq 2$ versus $>2$. All assessments were performed individually by two radiologists. The inter-rater agreement was calculated. The assessments were compared, and, in case of disagreement, consensus was reached in a joint reassessment that was used as the final result.

\section{Statistical analyses}

A professional statistician and a data manager from the clinical research centre at Uppsala University were involved in planning the study according to good clinical practice (GCP). In order to detect a critical change with a power of $80 \%$, a sample size of 44 participants (22 in the intervention group and 22 in the control group) that fulfilled the study protocol was required for a type I error of $5 \%$. Wilcoxon's signed-rank test was used to evaluate possible changes within a group between different test occasions. Spearman's rank test was used for correlation analysis of different test methods. The Mann-Whitney $U$ test and Fisher's exact test were used to compare data from the intervention group and the control group. When non-parametric tests were used, confidence intervals are presented to indicate the precision of the estimates. Interrater reliability was estimated using Cohen's Kappa (к). A value of $P<0.05$ was considered significant. All statistical analyses were performed using SAS version 9.1 (SAS Institute Inc., Carey, NC, USA).

\section{Results}

\section{Participants}

At 4 weeks post-stroke, 73 persons who had been identified with dysphagia one-week post-stroke were screened for persisting oropharyngeal swallowing dysfunction measured with TWST. Of these, 17 persons had regained normal swallowing function and were excluded, and 51 persons showed dysfunction, 11 of whom declined to participate. The remaining 40 participants were randomly assigned to either the intervention group $(n=$ 20, of who 9 were women; mean age 75 years, range 60 $85)$ or the control group $(n=20$, of who 6 were women; mean age 75 years, range 56-90) (See Fig. 4). Characterization of the participants and stroke lesion is shown in Table 1. For a further detailed description of the participants' characterization, see Additional file 2. After the treatment period, 32 participants $(80 \%)$ were alive and available for analysis, and at the 12-month follow-up 19 participants (47.5\%) were alive and available for analysis (see Fig. 3). The study was stopped somewhat prematurely due to a slow inclusion rate.

\section{Swallowing rate}

At the end of treatment, the intervention group and control group showed similar improvement in swallowing rate compared with baseline $(P<0.001$ and $P=0.001$, respectively; Table 2$)$ without any significant difference in improvement between the two groups $(P=0.133$; Table 2). At 12 months post-treatment, the swallowing rate had improved significantly more in the intervention group compared with the control group $(P=0.032$; Table 2). Individual line plots for changes in swallowing rate between time points are depicted in Fig. 5 .

\section{Lip force}

At the end of treatment, the intervention group showed an improvement in lip force $(P<0.001$; Table 2$)$, whereas the control group showed non-significant improvement in lip force $(P=0.079$; Table 2$)$. There was no significant between-group difference at the end of treatment $(P=0.066$; Table 2$)$. At 12 months posttreatment, lip force was significantly improved in the intervention group compared with the control group $(P=0.001$; Table 2). Individual line plots for changes in lip force between time points are depicted in Fig. 5.

\section{Videofluoroscopy}

At baseline, 32 of the 40 participants had available VFS. The remaining 8 were missing for logistical reasons. All 32 participants showed swallowing dysfunction at baseline, with PAS $>2$ in 53\% (intervention group, 50\%; controls, 57\%), premature spillage in $78 \%$ (intervention group, $72 \%$; controls, $86 \%$ ), and pharyngeal retention in $78 \%$ (intervention group, 83\%; controls, 71\%), see Table 3. Thus, there was good correspondence between the TWST score $<10 \mathrm{~mL} / \mathrm{sec}$ and the VFS findings. There was no significant difference in improvement between the two groups at any of the three test occasions 
Table 1 Baseline demographic and clinical characteristics

\begin{tabular}{|c|c|c|}
\hline Variable & Control group $n=20$ & Intervention group $n=20$ \\
\hline Age & 75 [56-90] & $75[60-85]$ \\
\hline \multicolumn{3}{|l|}{ Sex } \\
\hline Male & $14(70)$ & $11(55)$ \\
\hline Female & $6(30)$ & $9(45)$ \\
\hline \multicolumn{3}{|l|}{ Stroke type } \\
\hline Ischemic & $16(80)$ & $16(80)$ \\
\hline $\mathrm{ICH}$ & $3(15)$ & $4(20)$ \\
\hline Ischemic and ICH & $1(5)$ & - \\
\hline Left hemisphere & $6(30)$ & $7(35)$ \\
\hline Right hemisphere & $10(50)$ & $10(50)$ \\
\hline Supratentorial & $15(75)$ & $16(80)$ \\
\hline Infratentorial & $3(15)$ & $4(20)$ \\
\hline Supra- and infratentorial & $1(5)$ & - \\
\hline Lowered consciousness at hospital admission & $6(30)$ & $6(30)$ \\
\hline
\end{tabular}

Data are presented as $\mathrm{n}(\%)$ or mean [range]

Abbreviation: ICH Intracerebral hemorrhage

Table 2 Analysis of swallowing rate, lip force and VFS immediately after treatment and 12 months post-treatment

\begin{tabular}{|c|c|c|c|c|c|c|c|c|}
\hline \multirow[t]{2}{*}{ Outcome } & \multirow{2}{*}{$\begin{array}{l}\text { Control } \\
\text { Median } \\
\text { (ranges) }\end{array}$} & \multicolumn{2}{|l|}{$\begin{array}{l}\text { Within-group } \\
\text { differences }\end{array}$} & \multirow{2}{*}{$\begin{array}{l}\text { Intervention } \\
\text { Median (ranges) }\end{array}$} & \multicolumn{2}{|l|}{$\begin{array}{l}\text { Within-group } \\
\text { differences }\end{array}$} & \multirow{2}{*}{$\begin{array}{l}\text { Between-groups } \\
\text { differences } \\
P \text { value }\end{array}$} & \multirow{2}{*}{$\begin{array}{l}\text { No. subjects } \\
\text { Control/ } \\
\text { Intervention }\end{array}$} \\
\hline & & $(95 \% \mathrm{Cl})$ & $P$ value & & $(95 \% \mathrm{Cl})$ & $P$ value & & \\
\hline \multicolumn{9}{|l|}{ Swallowing rate ${ }^{a}$} \\
\hline Baseline & $5.1(0.0-9.5)$ & & & $5.3(0.0-9.2)$ & & & & $20 / 20$ \\
\hline $\begin{array}{l}\text { End of treatment } \\
\text { (5 weeks) }\end{array}$ & $9.2(1.8-13.4)$ & $3.6(1.0-5.8)$ & $0.001^{b}$ & $10.3(3.2-18.7)$ & $5.3(2.2-8.6)$ & $<0.001^{b}$ & $0.133^{c}$ & $14 / 18$ \\
\hline $\begin{array}{l}12 \text { months post- } \\
\text { treatment }\end{array}$ & $8.5(2.9-10.5)$ & $1.1(-1.0-6.6)$ & $0.164^{b}$ & $13.7(5.0-28.3)$ & $9.0(3.7-13.6)$ & $0.008^{b}$ & $0.032^{c}$ & $9 / 9$ \\
\hline \multicolumn{9}{|l|}{ Lip force } \\
\hline Baseline & $14.0(3.0-31.0)$ & & & $18.5(7.0-34.0)$ & & & & $14 / 18$ \\
\hline $\begin{array}{l}\text { End-of-treatment } \\
(5 \text { weeks) }\end{array}$ & $14.0(6.0-46.0)$ & $2(-1-10)$ & 0.079 & $27.0(11.0-59.0)$ & $7(3-13)$ & $<0.001$ & 0.066 & $14 / 18$ \\
\hline $\begin{array}{l}12 \text { months post- } \\
\text { treatment }\end{array}$ & $10.0(2.0-21.0)$ & $-1(-3-1)$ & 0.328 & $31.0(18.0-51.0)$ & $12(4-26)$ & 0.008 & 0.001 & $9 / 9$ \\
\hline \multicolumn{9}{|l|}{ VFS (PAS score) } \\
\hline Baseline & $6(1-8)$ & & & $3(1-8)$ & & & & 13/18 \\
\hline $\begin{array}{l}\text { End of treatment } \\
\text { (5 weeks) }\end{array}$ & $1(1-8)$ & $0.0(-6-6)$ & $0.524^{b}$ & $2(1-8)$ & $0.0(-2-0)$ & $0.219^{b}$ & 0.999 & $11 / 18$ \\
\hline $\begin{array}{l}12 \text { months post- } \\
\text { treatment }\end{array}$ & $1.5(1-8)$ & $0.0(-7-6)$ & $0.276^{\mathrm{b}}$ & $2(1-8)$ & $-0.5(-3-0)$ & $0.058^{b}$ & 0.912 & $8 / 9$ \\
\hline
\end{tabular}

Abbreviations: VFS videofluoroscopic evaluation of swallowing, PAS Penetration Aspiration Scale, Cl confidence interval, TWST timed water-swallow test

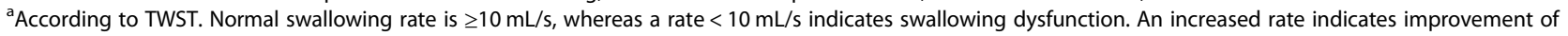
swallowing function

bWilcoxon signed-rank test

cMann Whitney U-test 


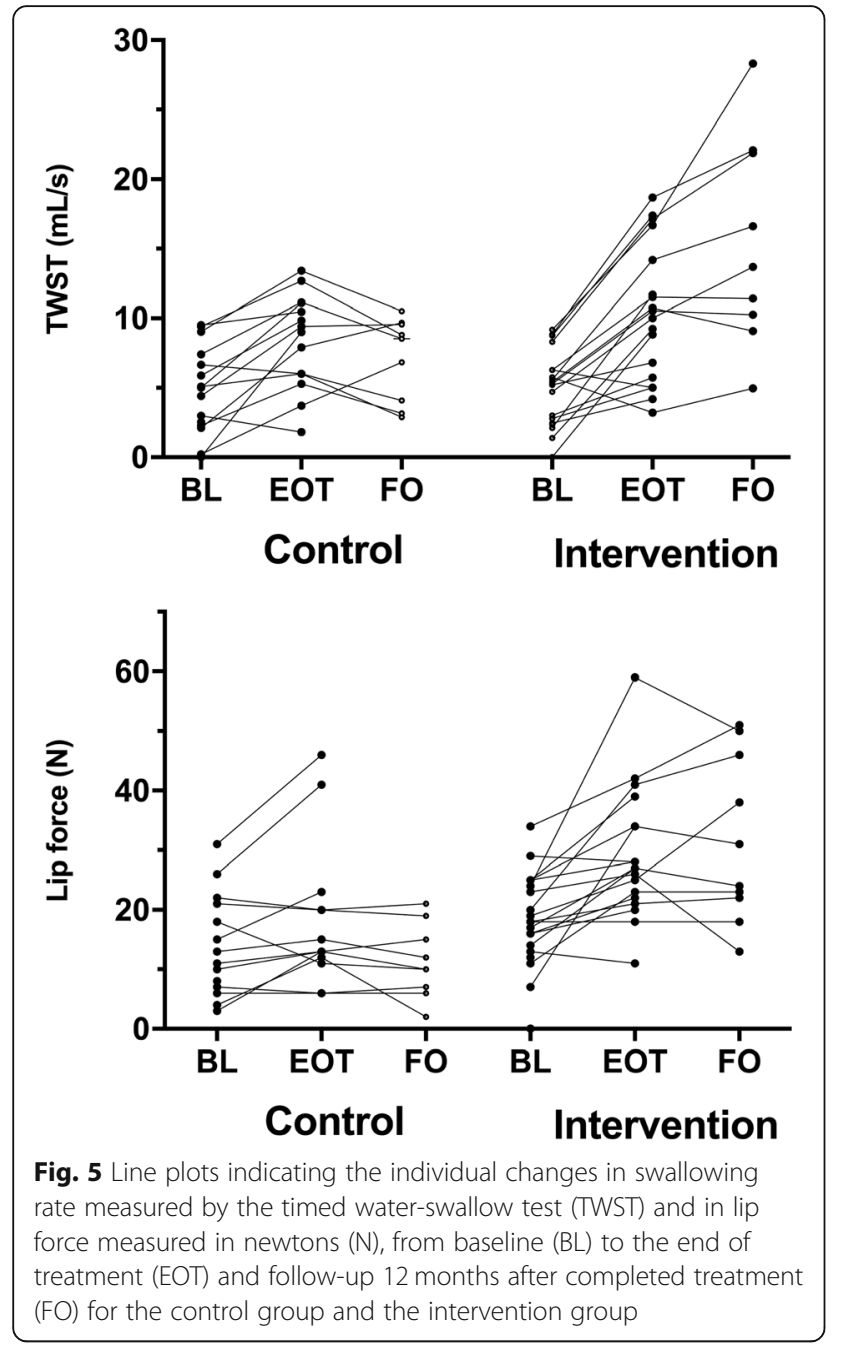

in any of the VFS parameters. The inter-rater reliability varied from near perfect agreement to perfect agreement for all measurements: premature spillage $(\kappa=0.93)$, pharyngeal retention $(\kappa=0.81)$, aspiration $(\kappa=1.00)$, and PAS $(\kappa=0.93)$.

\section{Adverse events}

No adverse events were registered in the intervention or the control group.

\section{Discussion}

This randomized controlled trial with blinded evaluators showed that oral neuromuscular training improved swallowing function in the long term among people with post-stroke dysphagia. While there were no significant between-group differences at the end of treatment in swallowing rate, lip force, or aspiration-penetration on VFS, the intervention group showed a significant improvement in swallowing rate and lip force at 12-month follow-up compared with the control group. These
Table 3 Swallowing dysfunction at baseline according to the VFS

\begin{tabular}{|c|c|c|c|}
\hline Baseline & $\begin{array}{l}\text { Control } \\
n=14\end{array}$ & $\begin{array}{l}\text { Intervention } \\
n=18\end{array}$ & $P$-value \\
\hline Premature spillage & & & $0.426^{a}$ \\
\hline Yes & $12(86)$ & $13(72)$ & \\
\hline No & $2(14)$ & $5(28)$ & \\
\hline Retention pharynx & & & $0.669^{a}$ \\
\hline Yes & $10(71)$ & $15(83)$ & \\
\hline No & $4(29)$ & $3(17)$ & \\
\hline Aspiration & & & $0.178^{b}$ \\
\hline Yes & $8(57)$ & $6(33)$ & \\
\hline No & $6(43)$ & $12(67)$ & \\
\hline PAS $>2$ & & & $0.688^{a}$ \\
\hline Yes & $8(57)$ & $9(50)$ & \\
\hline No & $6(43)$ & $9(50)$ & \\
\hline One or more dysfunction & & & NA \\
\hline Yes & $14(100)$ & $18(100)$ & \\
\hline No & $0(0)$ & $0(0)$ & \\
\hline
\end{tabular}

Data are given as $\mathrm{n}(\%)$

Abbreviations: VFS videofluoroscopy, PAS Penetration Aspiration Scale, NA Not Applicable

*Retention pharynx refers to retention in the vallecula or piriform sinus

${ }^{\text {a } F i s h e r ' s ~ e x a c t ~ t e s t ~}$

${ }^{\mathrm{b}}$ Chi-squared test

findings indicate the good effects of oral neuromuscular training as a treatment option for post-stroke dysphagia. There is, however, a need for larger randomized controlled trials to confirm our preliminary, positive longterm results.

Both the intervention and control groups showed significant within-group improvements in swallowing rate after the 5 -week training period, but there was no significant between-group difference. The nonsignificant between-group difference may be due to several factors. One is that both groups received active rehabilitation (toothbrush stimulation) during the treatment period and with positive outcome, indicating that both treatments had an immediate effect on swallowing. However, only the neuromuscular training was found to have a long-term effect. Another factor was the small sample size, indicating that there could be a lack of sufficient statistical power to generalise the conclusions of the findings. Yet another factor could be the spontaneous remission of swallowing dysfunction that is usually observed within 2-3 weeks after the stroke incident [16, 17]. In this study the participants were recruited 4 weeks after the stroke event, making it difficult to determine whether all the improvement in the swallowing rate was due to the therapeutic intervention or, to some extent, to spontaneous recovery. 
However, the lasting improvement in swallowing rate at 12 months post-treatment in the intervention group compared with the control group indicates that oral neuromuscular training may be more effective in triggering the reorganisation of the brain [18] than orofacial sensory-vibration stimulation alone. In contrast to orofacial sensory-vibration stimulation, oral neuromuscular training with an oral device aims to both stimulate sensory input and strengthen the facial, oral, and pharyngeal muscles [13].

Exercised-based approaches to swallowing rehabilitation have been shown to not only improve swallowing function in people with dysphagia, but to improve oral diet and reduce aspiration and dependence on tube feeding [19-22]. Furthermore, exercises to strengthen the swallowing musculature improve swallowing and reduce dysphagia-related comorbidities [19, 21]. In the present study, swallowing function was assessed and improved after oral neuromuscular training, indicating that combined sensorimotor exercise might be a more beneficial swallowing rehabilitation method in the long term than sensory stimulation alone. However, we did not evaluate the effect of oral neuromuscular training on dysphagiarelated comorbidities such as malnutrition, dehydration, or pneumonia. Further studies should include these aspects and include swallowing-related quality of life as outcomes when evaluating oral neuromuscular training effects on swallowing dysfunction. Thus, in order to evaluate the full benefit of oral neuromuscular training as a swallowing rehabilitation method.

Improvement in lip force was observed in the intervention group both after 5 weeks of oral neuromuscular training and at the 12-month follow-up, whereas no significant improvement was observed in the control group at any time. A significant between-group difference was observed only at the 12-month follow-up. These findings, combined with the improvements in swallowing rate, seem to support the finding of previous studies $[8,13]$ in which oral neuromuscular training improved both swallowing rate and lip force.

When used to identify swallowing dysfunction, results from TWST proved to have excellent correlation with findings from VFS, in accordance with a previous study [14]. All participants showed dysfunction, either with penetration/aspiration of contrast medium entering the airway and causing unsafe swallowing at baseline, or with premature spillage and/or pharyngeal residue revealing ineffective pharyngeal swallowing. It is essential to have valid and reliable screening tools in clinical settings since gold standard assessments (VFS and videoendoscopy) are not always available or feasible to implement due to both social and economic conditions, as well as the existing health policies. The advantage of TWST is that the tool can easily be used as a quantifiable measure of treatment effect $(\mathrm{mL} / \mathrm{s}$ before and after treatment). The disadvantage is that the tool only assesses the swallowing function using liquid bolus. Other potential screening tools for swallowing assessment that includes tests of semi-solid and solid texture is, e.g., the Gugging Swallowing Screen (GUSS) [23] or the volume-viscosity swallow test (V-VST) [24].

The present study is the first randomized controlled trial of oral neuromuscular training with an oral device as post-stroke dysphagia treatment and in which blinded assessors were used at the end of treatment and at 12month follow-up. However, it does have some methodological issues. A common challenge with clinical trials, including this study, is to recruit enough participants to obtain acceptable statistical power at follow-up, after drop-out. The number of participants included at baseline almost reached the number targeted by the power analyses, but many participants were lost at follow-up due to mortality, PEG, recurrent stroke, or because they declined continued participation (see Fig. 3 and Additional file 2). Another limitation is that the randomization to either the intervention or control group was based on TWST results and not on VFS findings. Since the randomization procedure did not take into account important prognostic factors at baseline, an uneven distribution of aspiration at baseline (33\% in the intervention group and 57\% among controls) might have contributed to the lack of significant outcome on differences in improvement according to VFS. It might have been easier to improve in PAS in the control group with higher frequency of aspiration at baseline than in the intervention group. Thus, an objective finding on the severity of swallowing dysfunction may be an important prognostic factor in the participants' ability to improve the impaired function, and should be considered at randomisation.

\section{Conclusions}

Oral neuromuscular training in participants with impaired swallowing rate after stroke had no significant effect on swallowing rate immediately after the 5-week training but resulted in long-term improvement in swallowing function. TWST findings corresponded with VFS findings and TWST therefore appears to be a feasible method with which to identify persons with swallowing dysfunction after stroke. Larger randomized controlled trials are required to confirm the preliminary positive long-term results on swallowing function in this study.

\section{Supplementary Information}

The online version contains supplementary material available at https://doi. org/10.1186/s12883-020-01980-1.

Additional file 1 
Additional file 2. Participants' characteristics

\section{Abbreviations}

TWST: Timed water-swallow test; VFS: Videofluoroscopy; PAS: Penetrationaspiration scale

\section{Acknowledgements}

We wish to thank speech language pathologist Heléne Westerlund and research nurses Rolf Backlund and Erika Strålberg for clinical support and biostatistician Lisa Wernroth, Uppsala Clinical Research Centre (UCR), who was responsible for statistical analyses. We also wish to thank research engineer Magnus Johansson, MSc, for his contribution of the images used in this article.

\section{Authors' contributions}

$\mathrm{MH}, \mathrm{PH}, \mathrm{EL} J$ and PW contributed to the conception and design of the work. $\mathrm{PH}, \mathrm{MH}, \mathrm{BL}, \mathrm{EL} J$ and PW contributed to the acquisition of data. PH and Lisa Wernroth, certified statistician (acknowledged) was responsible for the statistical analyses. PH and $\mathrm{MH}$ drafted the first version of the manuscript. All authors critically revised the manuscript for important intellectual content and gave their final approval for publication.

\section{Funding}

This study was supported by the Gävleborg and Örebro County Region [852221 and 156941], the Regional Research Council in Uppsala University, the CAPIO research foundation, the Swedish Heart and Lung Foundation, the County council of Västerbotten, the Swedish Stroke Association and the Northern Swedish Stroke Association. The funding bodies played no role in the design of the study and collection, analysis, and interpretation of data and in writing the manuscript. Open Access funding provided by University of Umea.

\section{Availability of data and materials}

The datasets generated and/or analysed during the current study are not publicly available due to institutional restrictions but are available from the corresponding author on reasonable request.

\section{Ethics approval and consent to participate}

The study was performed according to the Helsinki Declaration, and was approved by the Uppsala Regional Ethics Review Board at Uppsala University, Sweden (Dnr 2004 M-435). Informed written and verbal consent was obtained from all the participants as approved by the Regional Ethics Review Board. The consent was documented in their medical records. The person illustrating the measurement of lip-force in Fig. 2 is not a patient and has given written consent for publication of the image.

\section{Consent for publication}

The person illustrating the measurement of lip-force in Fig. 2 is not a patient and has approved publication of the image.

\section{Competing interests}

The authors declare that they have no competing interests.

\section{Author details}

'Department of Odontology, Oral and Maxillofacial Radiology, Umeå University, SE-90187 Umeå, Sweden. ${ }^{2}$ Umeå Stroke Center, Department of Public Health and Clinical Medicine, Umeå University, Umeå, Sweden. ${ }^{3}$ Speech \& Swallowing Centre, Department of Otorhinolaryngology, Hudiksvall Hospital, Hudiksvall, Sweden. ${ }^{4}$ Centre for Research \& Development, Uppsala University/County Council of Gävleborg, Gävle, Sweden. ${ }^{5}$ Department of Radiology, Hudiksvall Hospital, Hudiksvall, Sweden. ${ }^{6}$ Department of Clinical Sciences, Karolinska Institutet, Danderyd University Hospital, Stockholm, Sweden.

Received: 6 March 2020 Accepted: 28 October 2020

\section{Published online: 07 November 2020}

\section{References}

1. Martino R, Foley N, Bhogal S, Diamant N, Speechley M, Teasell R. Dysphagia after stroke: incidence, diagnosis, and pulmonary complications. Stroke. 2005;36(12):2756-63. https://doi.org/10.1161/01.STR.0000190056.76543.eb.
2. Castillo Morales RC, Brondo JJ, Haberstock B. Die orofaziale Regulationstherapie. 1st ed. München: Richard Pflaum Verlag $\mathrm{GmbH} \& \mathrm{Co}$; 2019. p. 21-188.

3. Hiraba H, Yamaoka M, Fukano M, Fujiwara T, Ueda K. Increased secretion of salivary glands produced by facial vibrotactile stimulation. Somatosens Mot Res. 2008;25(4):222-9.

4. Hagg M, Larsson B. Effects of motor and sensory stimulation in stroke patients with long-lasting dysphagia. Dysphagia. 2004;19(4):219-30.

5. Thuer $U$, Ingervall B. Effect of muscle exercise with an oral screen on lip function. Eur J Orthod. 1990;12(2):198-208.

6. Hagg M, Olgarsson M, Anniko M. Reliable lip force measurement in healthy controls and in patients with stroke: a methodologic study. Dysphagia. 2008;23(3):291-6.

7. Perkins RE, Blanton PL, Biggs NL. Electromyographic analysis of the "baccinator mechanism" in human beings. J Dent Res. 1977;56(7):783-94.

8. Hagg M, Anniko M. Influence of lip force on swallowing capacity in stroke patients and in healthy subjects. Acta Otolaryngol. 2010;130(11):1204-8.

9. Hagglund P, Hagg M, Wester P, Levring JE. Effects of oral neuromuscular training on swallowing dysfunction among older people in intermediate care-a cluster randomised, controlled trial. Age Ageing. 2019;48(4):533-40.

10. Nathadwarawala KM, Nicklin J, Wiles CM. A timed test of swallowing capacity for neurological patients. J Neurol Neurosurg Psychiatry. 1992;55(9): 822-5.

11. Nathadwarawala KM, McGroary A, Wiles CM. Swallowing in neurological outpatients: use of a timed test. Dysphagia. 1994;9(2):120-9.

12. Hughes TA, Wiles CM. Clinical measurement of swallowing in health and in neurogenic dysphagia. QJM. 1996;89(2):109-16.

13. Hagg M, Anniko M. Lip muscle training in stroke patients with dysphagia. Acta Otolaryngol. 2008;128(9):1027-33.

14. Wu MC, Chang YC, Wang TG, Lin LC. Evaluating swallowing dysfunction using a 100-ml water swallowing test. Dysphagia. 2004;19(1):43-7.

15. Rosenbek JC, Robbins JA, Roecker EB, Coyle JL, Wood JL. A penetrationaspiration scale. Dysphagia. 1996;11(2):93-8.

16. Mann G, Hankey GJ, Cameron D. Swallowing function after stroke: prognosis and prognostic factors at 6 months. Stroke. 1999;30(4):744-8.

17. Paciaroni M, Mazzotta G, Corea F, Caso V, Venti M, Milia P, et al. Dysphagia following stroke. Eur Neurol. 2004;51(3):162-7.

18. Hamdy S, Rothwell JC, Aziz Q, Singh KD, Thompson DG. Long-term reorganization of human motor cortex driven by short-term sensory stimulation. Nat Neurosci. 1998;1(1):64-8.

19. Crary MA, Carnaby GD, LaGorio LA, Carvajal PJ. Functional and physiological outcomes from an exercise-based dysphagia therapy: a pilot investigation of the McNeill dysphagia therapy program. Arch Phys Med Rehabil. 2012; 93(7):1173-8.

20. Speyer R, Baijens L, Heijnen M, Zwijnenberg I. Effects of therapy in oropharyngeal dysphagia by speech and language therapists: a systematic review. Dysphagia. 2010;25(1):40-65.

21. Robbins J, Gangnon RE, Theis SM, Kays SA, Hewitt AL, Hind JA. The effects of lingual exercise on swallowing in older adults. J Am Geriatr Soc. 2005; 53(9):1483-9.

22. Sura L, Madhavan A, Carnaby G, Crary MA. Dysphagia in the elderly: management and nutritional considerations. Clin Interv Aging. 2012;7:287-98.

23. Trapl M, Enderle P, Nowotny M, Teuschl Y, Matz K, Dachenhausen A, et al. Dysphagia bedside screening for acute-stroke patients: the Gugging swallowing screen. Stroke. 2007;38(11):2948-52.

24. Rofes L, Arreola V, Mukherjee R, Clave P. Sensitivity and specificity of the eating assessment tool and the volume-viscosity swallow test for clinical evaluation of oropharyngeal dysphagia. Neurogastroenterol Motil. 2014; 26(9):1256-65.

\section{Publisher's Note}

Springer Nature remains neutral with regard to jurisdictional claims in published maps and institutional affiliations. 